\title{
Research into magnetic guidance technology for directional drilling in SAGD horizontal wells
}

\author{
Gao Deli*, Diao Binbin, Wu Zhiyong and Zhu Yu \\ State Key Laboratory of Petroleum Resource \& Prospecting, MOE Key Laboratory of Petroleum Engineering, China \\ University of Petroleum, Beijing 102249, China
}

(C) China University of Petroleum (Beijing) and Springer-Verlag Berlin Heidelberg 2013

\begin{abstract}
SAGD horizontal wells are used to enhance oil recovery from heavy oil reservoirs. This technology requires precise separation between the production well and the injection well to ensure the efficient drainage of the reservoir. By studying the attitude of the downhole probe tube and the production well trajectory, an algorithm is proposed for eliminating ferromagnetic interference while drilling injection wells. A high accuracy filter circuit has been designed to correct the detected magnetic signals, which are ultra-weak, frequency-instable, and narrow-band. The directional drilling magnetic guidance system (DD-MGS) has been developed by integrating these advanced techniques. It contains a sub-system for the ranging calculation software, a magnetic source, a downhole probe tube and a sub-system for collecting $\&$ processing the detected signals. The DD-MGS has succeeded in oilfield applications. It can guide the directional drilling trajectory not only in the horizontal section but also in the build section of horizontal injection wells. This new technology has broad potential applications.
\end{abstract}

Key words: Heavy oil, SAGD horizontal wells, directional drilling, magnetic guidance system

\section{Introduction}

Thermal oil recovery is one of the most effective means for the production of heavy oil and there are several effective methods. These include steam soaking, steam drive, steam assisted gravity drainage (SAGD) (Corbett, 1997) and in-situ combustion, of which SAGD is the most effective (Yang et al, 2010). The SAGD technique is often used for recovering ultra heavy oil and its main well patterns are vertical-horizontal well combinations (Malik et al, 2011; Bao et al, 2012) and twin-horizontal wells (Mendoza et al, 1999). Typical SAGD horizontal wells drilled in the Karamay oilfield in western China have a specified separation of $5.0 \pm 0.5$ meters between the production well and the injection well (Lin et al, 2009). Due to ferromagnetic interference from the completion tubular string (casing and screen pipe) and the accumulated errors, conventional inclinometers cannot be used to guide the directional drilling trajectory in the SAGD horizontal wells.

In order to meet the field requirement, Vector Magnetics LLC in USA developed the Magnetic Guidance Tool (MGT) (Kuckes et al, 1996; Grills, 2002) and the Rotating Magnet Ranging System (RMRS) (Vandal et al, 2001; Rach, 2004; AlKhodhori et al, 2008; Loparev, 2008; Oskarsen et al, 2009). The MGT was the first method proposed for directional drilling in SAGD horizontal wells. It has been utilized to drill over $95 \%$ of the SAGD horizontal wells in existence

*Corresponding author. email: gaodeli@cup.edu.cn

Received June 20, 2013
(Vandal et al, 2004). The RMRS has the advantages of high precision and wide range. However, its maximum working temperature is only $85^{\circ} \mathrm{C}$, its operations are complicated and its sensors are susceptible to the ferromagnetic interference. The RMRS system has been proven to be effective for well intersection operations in which a vertical well is intersected by a horizontal well (Qiao et al, 2007; Gong and Duan, 2006; $\mathrm{Hu}$ and Chen, 2008; Dong et al, 2008). However, the RMRS and the MGT need a lot of detected data, and the magnetic source or the probe tube needs to be run into the production well, so that they are not suitable for the applications in relief-well intersection operations and collision avoidance in cluster-well engineering. Therefore, Vector Magnetics LLC developed the Single Wire Guidance Tool (Mallary et al, 1998) and Wellspot Tool (Jones et al, 1987; Grace et al, 1988; Olberg et al, 1991; Tarr et al 1992; Roes et al, 1998). The authors are also studying new electromagnetic guidance systems for directional drilling ( $\mathrm{Li}$ et al, 2012; Diao and Gao, 2013), which have not yet been applied in an oilfield.

The magnetic guidance technology of SAGD horizontal well drilling has been developing rapidly in China recently. Yang et al (2010) introduced the directional drilling technology in SAGD horizontal wells and its applications. Gao, Diao, Sun and other researchers have done much investigation into the theory and hardware of RMRS and MGT (Diao and Gao, 2011; Diao et al, 2011a; 2011b; Sun et al, 2011). We have developed a magnetic guidance system for directional drilling (DD-MGS) in SAGD horizontal wells by investigating magnetic ranging theory and methods 
for collecting ultra-weak magnetic signals. The magnetic guidance technology for directional drilling in SAGD horizontal wells has been developed with the DD-MGS, and it has been tested and applied successfully in the Fengcheng heavy oil field in Xinjiang, China.

\section{Composition of the DD-MGS}

The DD-MGS is mainly composed of a magnetic sub (drill-stem connector), a downhole probe tube, a signal collecting and processing system, and ranging calculation software. The DD-MGS can be used to measure the distance between two adjacent wells and precisely control the trajectory of complex-structure wells. As shown in Fig. 1, the magnetic sub installed near the bit is composed of several permanent magnets and a short non-magnetic pipe. When rotating with the drill tool, the magnetic sub will produce an alternating magnetic field, which is the magnetic source of the DD-MGS. The downhole probe tube is used to measure the alternating magnetic field and the measured signal data is then transmitted through the cable to the data collecting and processing system on the surface. The downhole probe tube has a sensor package composed of a tri-axial magnetometer, a tri-axial acceleration sensor and a tri-axial alternating magnetic field sensor. With these components, the downhole probe tube can collect the signals including the attitude of the probe tube and the induction density of the alternating magnetic field. Then the signal data can be transmitted to a computer in the situ office where the ranging calculation software can be used to analyze the data. After determining the relative positions and directions of the two wells based on these measurements and calculations, the well trajectory can be accurately controlled as required.

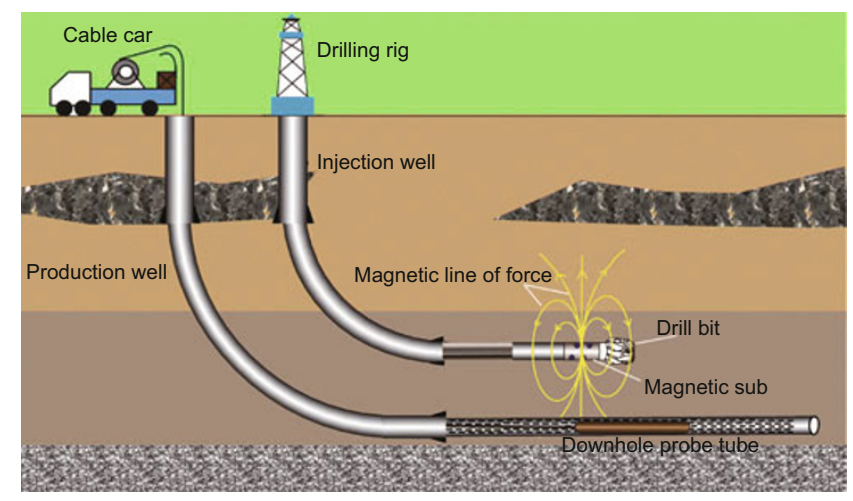

Fig. 1 The DD-MGS in SAGD horizontal wells

\section{Working principle of the DD-MGS}

When the magnetic sub is rotating above the downhole probe tube, the $Z$ axis magnetic induction density $\left(B_{Z}\right)$ detected by the magnetic vector sensors is shown in Fig. 2. The radial distance between the downhole magnetic sub and the probe tube is twice the distance of the magnetic sub covered from one wave peak to the nearest trough along the $Z$ axis or equal to the distance of the magnetic sub covered from one wave peak to the adjacent other wave peak along the $Z$ axis.

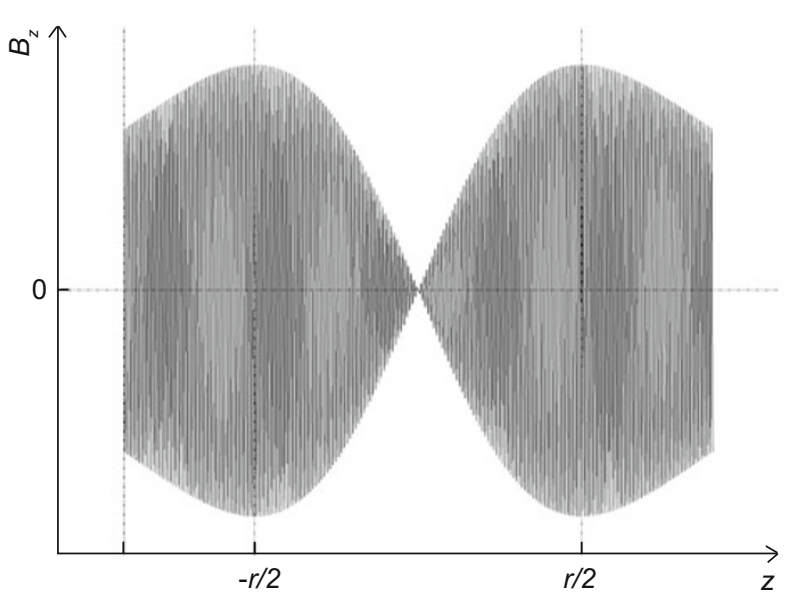

Fig. 2 Variation curve of $B_{z}$ with $z$

As shown in Fig. 3, the unit vector $\hat{m}$ stands for the direction of the equivalent magnetic moment of the magnetic sub at a certain time and the unit vector $\hat{h}$ for the direction of the high side of the wellbore. The unit vector $\hat{w}$ stands for the trajectory extension direction of the production well. According to the right-hand rule, the unit vector $\hat{v}$ is orthogonal to the unit vector $\hat{h}$ and the unit vector $\hat{w} . A_{h r}$ stands for the angle between the unit vector $\hat{h}$ and the unit vector $\hat{r}$. The value of $A_{h r}$ can be used to calculate the relative direction between the two SAGD horizontal wells.

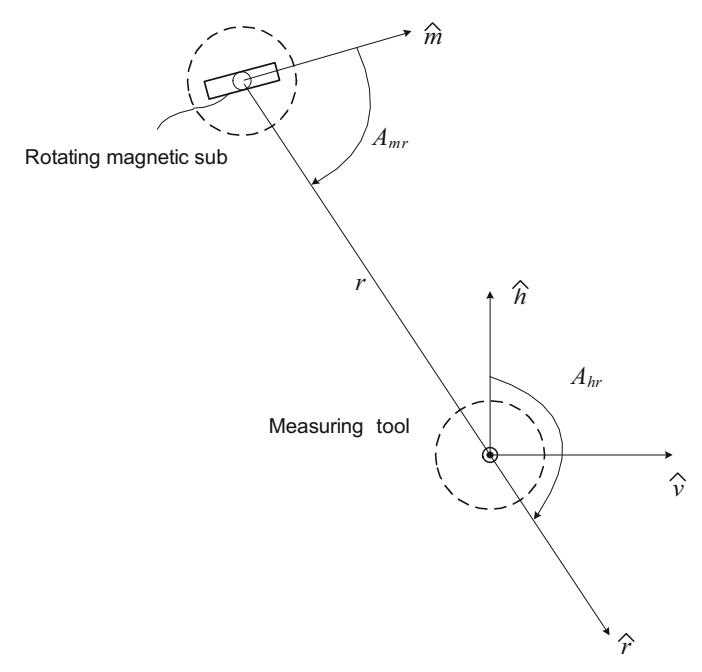

Fig. 3 Ranging calculation model for SAGD horizontal wells

When the center of the magnetic sub points at the center of the downhole probe tube perpendicularly, the amplitudes of $B_{h}$ and $B_{v}$ can be expressed as follows:

$$
\begin{aligned}
& \left|B_{h}\right|=\frac{m}{4 \pi r^{3}} \sqrt{4 \cos ^{2}\left(A_{h r}\right)+\sin ^{2}\left(A_{h r}\right)} \\
& \left|B_{v}\right|=\frac{m}{4 \pi r^{3}} \sqrt{4 \sin ^{2}\left(A_{h r}\right)+\cos ^{2}\left(A_{h r}\right)}
\end{aligned}
$$

Then, the following equation can be derived from Eqs. (1) and (2): 


$$
\cos \left(2 A_{h r}\right)=\frac{5}{3} \frac{\left|B_{h}\right|^{2}-\left|B_{v}\right|^{2}}{\left|B_{h}\right|^{2}+\left|B_{v}\right|^{2}}
$$

where $m$ is the value of the equivalent magnetic moment of the magnetic sub; $B_{h}$ and $B_{v}$ can be deduced from the magnetic induction intensity through coordinate transformation.
Because of the ferromagnetic interference induced by the downhole casing and screen pipe, the amplitudes of the magnetic induction intensity detected by the probe tube should be corrected in actual applications. Thus, the $A_{h r}$ can be calculated from Eq. (3). The calculation flow is shown in the Fig. 4.

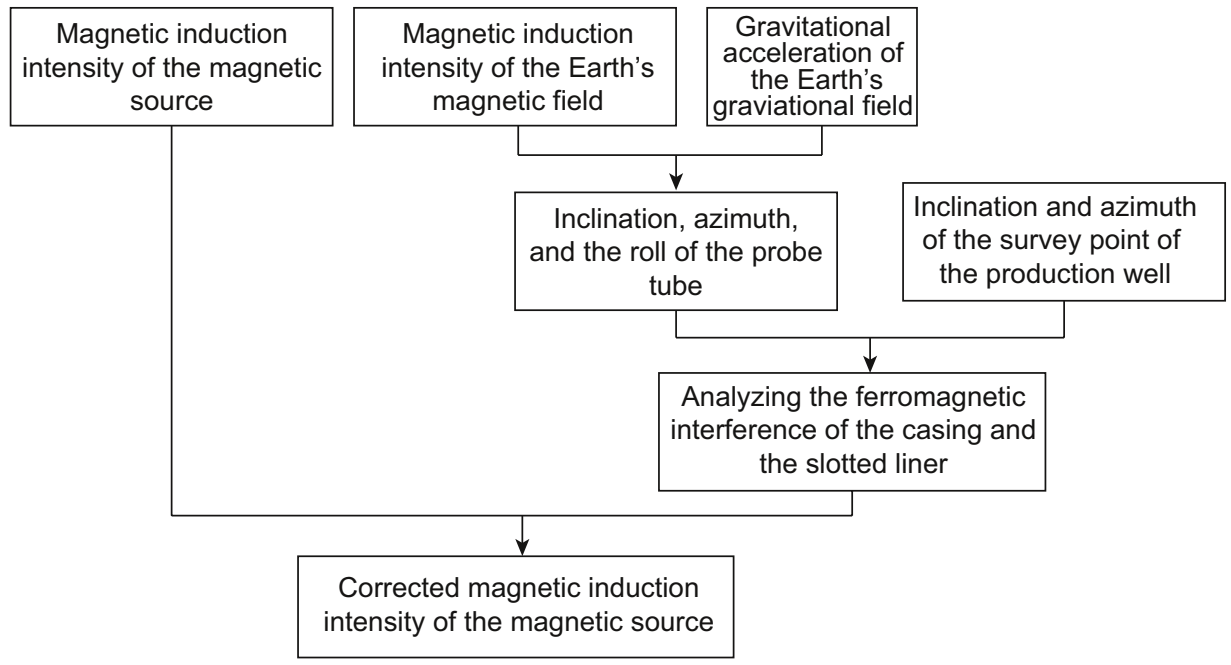

Fig. 4 Calculation flow chart for eliminating the ferromagnetic interference

\section{Key hardware design}

\subsection{Design of the collecting system for weak signals}

Based on a study of the theory and practical measurements, the characteristics of the magnetic field signal to be detected can be described as follows:

a) The signal is ultra weak because the magnetic field strength decreases proportionally to $1 / r^{3}$ while the downhole probe tube is moving away from the magnetic source, where $r$ is the distance between the downhole magnetic sub and the probe tube.

b) The magnetic field signal produced is characterized by ultra-low frequency, narrow bandwidth, and frequencyinstability. The frequency of the magnetic source changes from 2 to $5 \mathrm{~Hz}$ with the rotating speed of the drill bit.

c) There is plenty of noise mixed with the magnetic field signal. When the distance between the downhole magnetic sub and the probe tube exceeds $30 \mathrm{~m}$, the weak signal will be buried by the noise.

Based on the characteristics of the detected signals, the signal collecting system has been designed as shown in Fig. 5 .

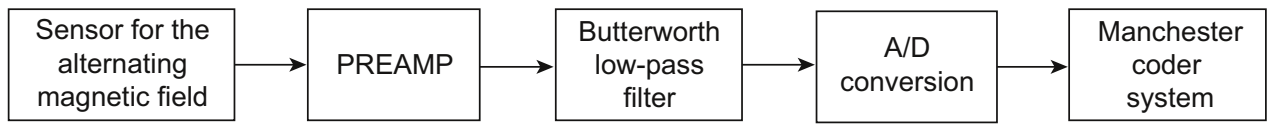

Fig. 5 Signal collecting system

The tri-axis magnetic field sensor transforms the magnetic signal into an electronic signal. The preamplifier circuit and Butterworth low-pass filter can reduce the noise and obtain the weak signal. The A/D converter converts the analog signal into the digital signal. The Manchester coder system can encode and transmit the data to the signal collecting and processing system located at the situ office.

The magnetic field signal from the tri-axial alternating magnetic field sensor must be the superposition of the Earth's magnetic field and the alternating magnetic field of the magnetic sub, so that the first preamplifier circuit is especially important. The first preamplifier circuit has been designed as shown in Fig. 6. It is a high-gain positive-feedback and multiple-pole high-pass amplifier circuit, which can eliminate the effect of the Earth's magnetic field and amplify the effective magnetic signal. In order to collect the signal from 2.0 to $5.0 \mathrm{~Hz}$, a 6-step Butterworth low-pass filter (Fig. 7) is used to reduce the noise and improve the signal noise ratio (SNR) .

\subsection{Design of the downhole probe tube}

The downhole probe tube is run into the production well through an armored cable and the wellbore length is limited to $3,000 \mathrm{~m}$. The required working conditions of the DD-MGS are as follows:

a) The temperature is under $85^{\circ} \mathrm{C}$.

b) The pressure is under $100 \mathrm{MPa}$.

c) The cable resistance and the cable capacitance are less than or equal to $100 \Omega$ and $0.5 \mu \mathrm{F}$, respectively.

The DD-MGS has the following main functions: 


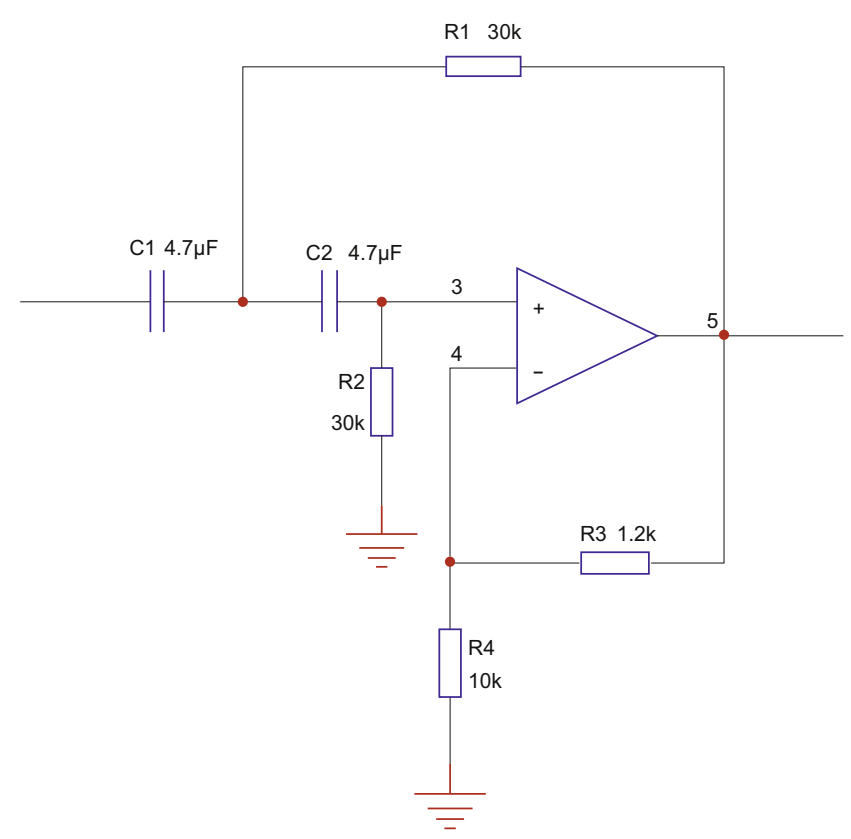

Fig. 6 Front $\mathrm{AC}$ amplifier circuit

a) The system can be used to measure the inclination and azimuth of the downhole probe tube and then to calculate its attitude.

b) The system can be used to detect the three-axis magnetic induction density from the magnetic source in realtime.

c) The system can be used to measure the hardware temperature so as to evaluate the error produced by the temperature.

d) The system can be used to transmit the downhole data to a computer at the situ office to calculate the separation distances using the ranging calculation software.

The Model 544 is an integrated chip, which contains the three-axis accelerometer, the three-axis magnetometer and the temperature sensor. The size of the Model 544 is $0.75^{\prime \prime} \times 0.80^{\prime \prime} \times 4.6^{\prime \prime}$ to make it suitable for the downhole probe tube. The combination of Model 544 with the alternating magnetic field sensor can meet the requirements of the downhole probe tube.

The structure of the downhole probe tube is shown in Fig. 8. It is composed of the following parts:

a) The circuit for signal conversion, which can transform the signal into a suitable pattern for long line transmission.

b) The circuit for processing the tri-axial alternating magnetic signal, which can amplify and filter the signal from the magnetic sub and transform the magnetic signal into digital data.

c) The sensor for measuring the alternating magnetic field.

d) Model 544 chip.

The product picture of the downhole probe tube is shown in Fig. 9.

\section{Analysis of the field applications}

In 2012, the DD-MGS was tested in directional drilling for several SAGD horizontal wells in the Fengcheng oil field in Xinjiang. But the DD-MGS was not utilized until directional drilling reached the horizontal section of the

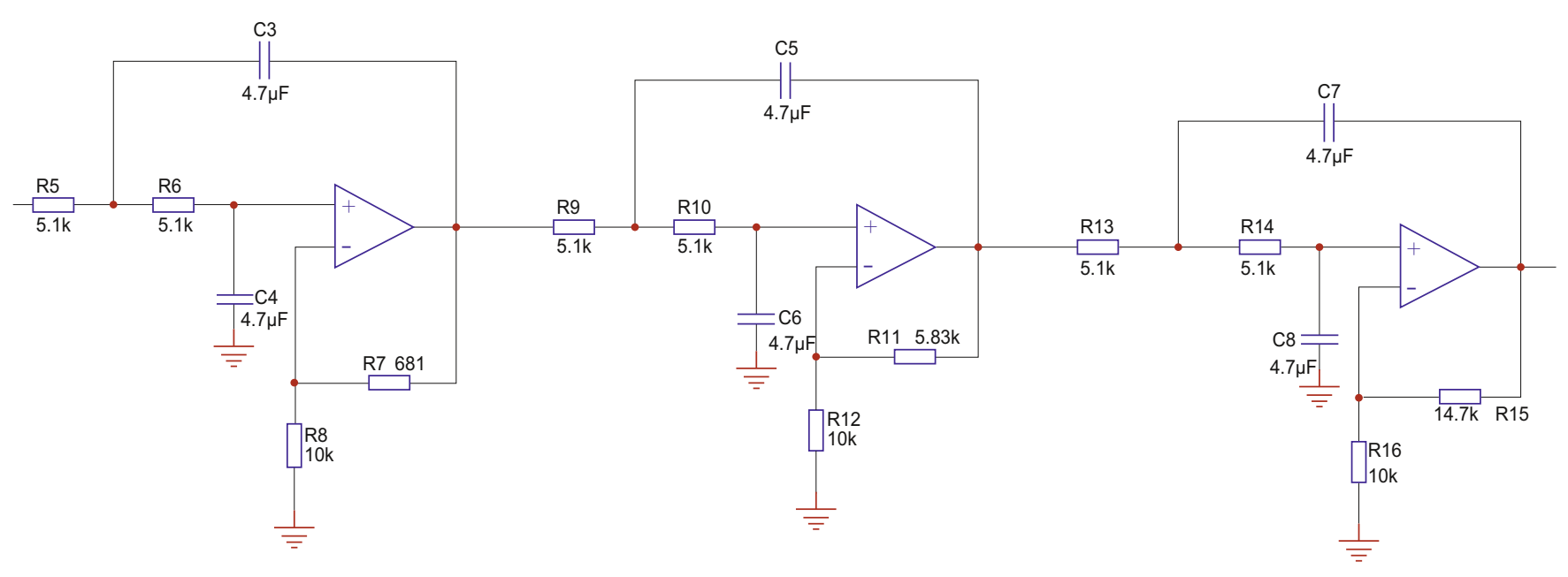

Fig. 7 Positive feedback type low-pass filter

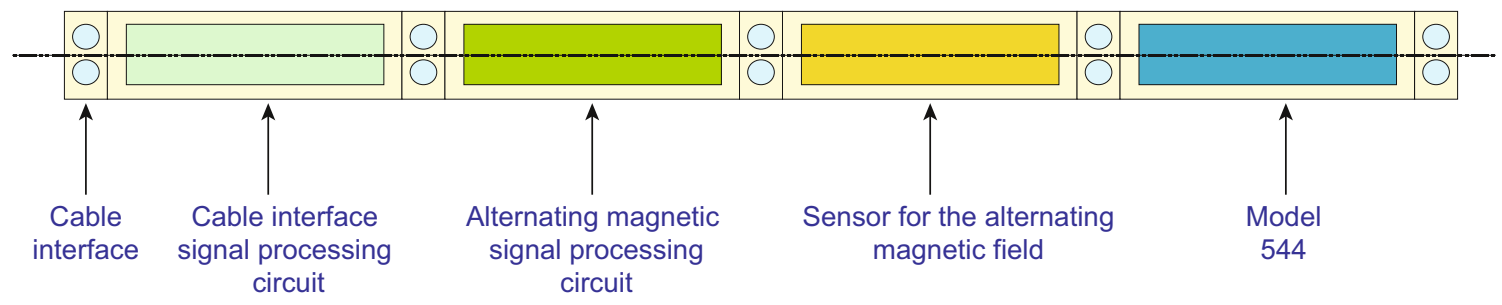

Fig. 8 Structure of the downhole probe tube 
production well. This situation makes it difficult for the well trajectory control to hit the target vertically in the horizontal window. Because of this disadvantage, the distance between the production well and the injection well must be adjusted in about the first $100 \mathrm{~m}$ of the horizontal section, as shown in Fig. 10.
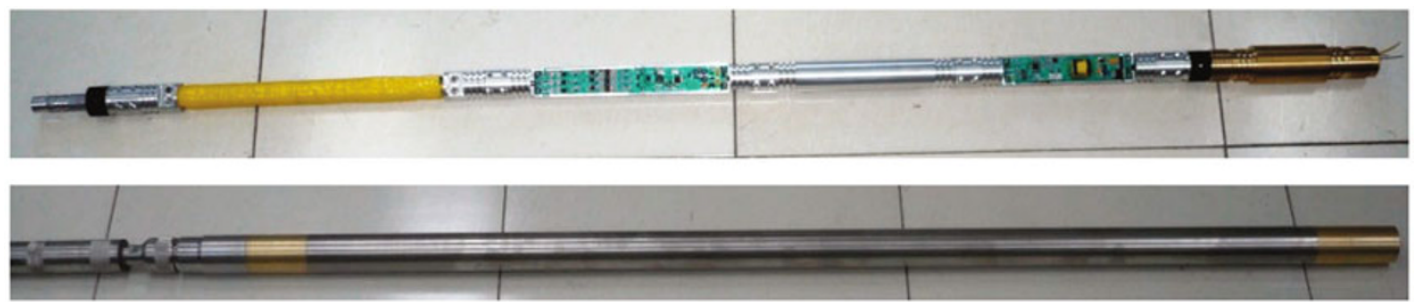

Fig. 9 Product picture of the downhole probe tube

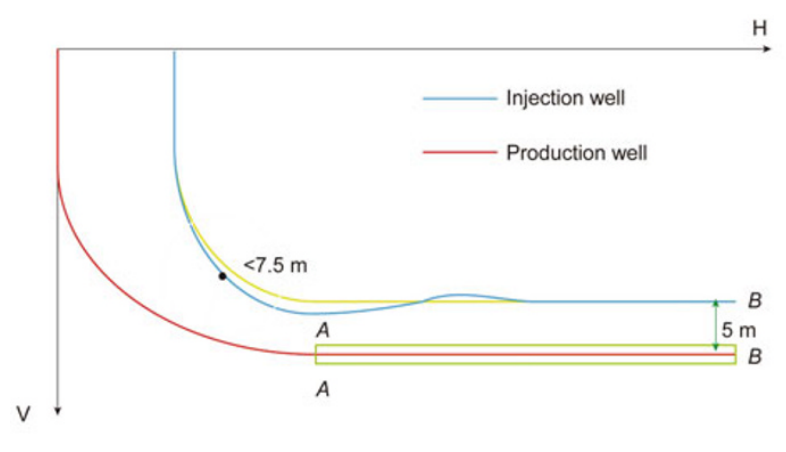

(a) Vertical section

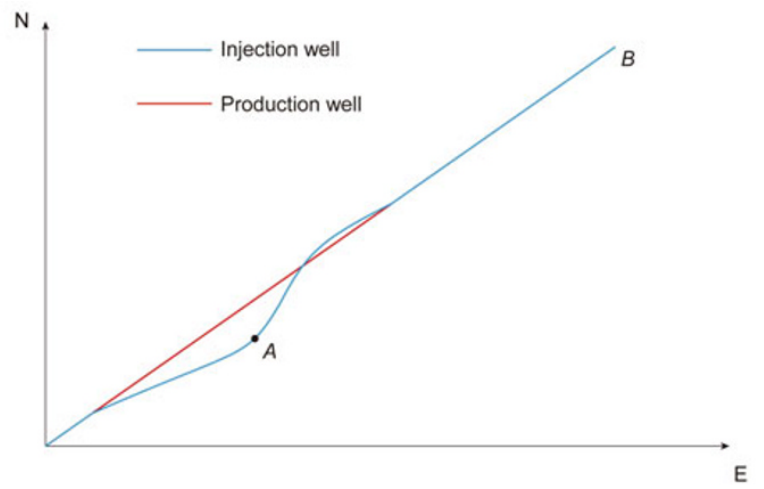

(b) Horizontal projection

Fig. 10 Profile of the injection well and the production well

In 2013, the improved DD-MGS had excellent performance in the directional drilling control of SAGD horizontal wells (Well I and Well P) in the Xingjiang heavy oil field. The DD-MGS can be used not only in the horizontal section but also in the build section, which is a breakthrough in directional drilling technology for SAGD horizontal wells. The uncertainty analysis of the well trajectory is shown in Fig. 11. Due to the conventional accumulated errors of the well trajectory survey, there is a collision risk in directional drilling in the horizontal section of the injection well. The production well (Well P) has been completed already by casing running shown in Fig. 12. The distance between the injection well (Well I) and the production well (Well P) is only $5 \mathrm{~m}$, so ferromagnetic interference from the casing and the slotted liner will disturb the measurement while drilling (MWD) in directional drilling.

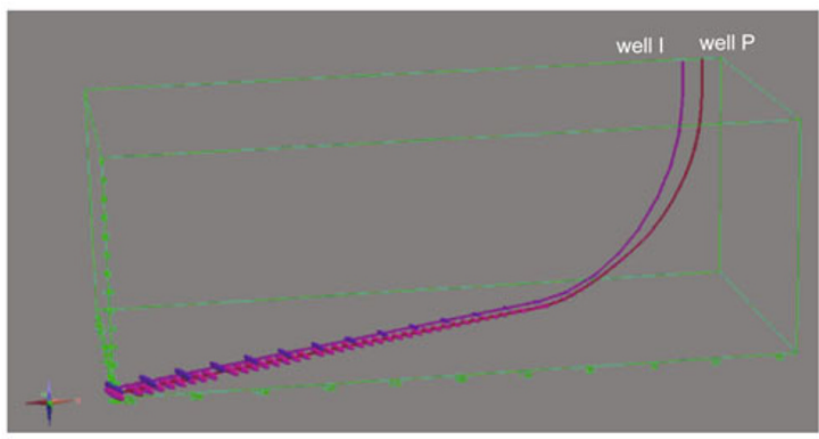

Fig. 11 The trajectory uncertainty analysis of Well I and Well P

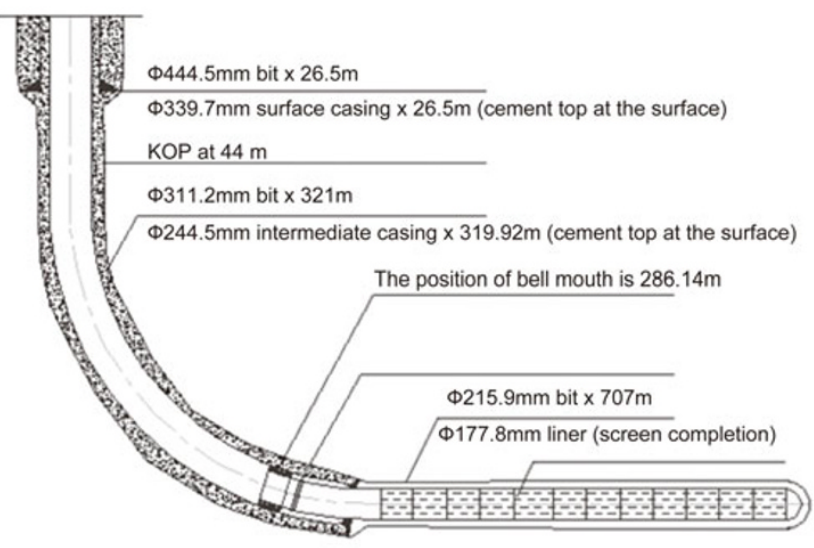

Fig. 12 Configuration of Well $\mathrm{P}$

In order to improve the precision of injection well trajectory control, the DD-MGS can be used to detect and guide the directional drilling after an inclination of 60 degrees. The results obtained by measurement $\&$ calculation while drilling are shown in Fig. 13.

As shown in Fig. 13, the accuracy of hitting the target in the horizontal window is high enough to meet the requirement by using the DD-MGS in the build section of the horizontal injection well. Thus, the quality and efficiency of directional drilling operation for horizontal section of the injection well can be improved greatly, as shown in Fig. 14. 

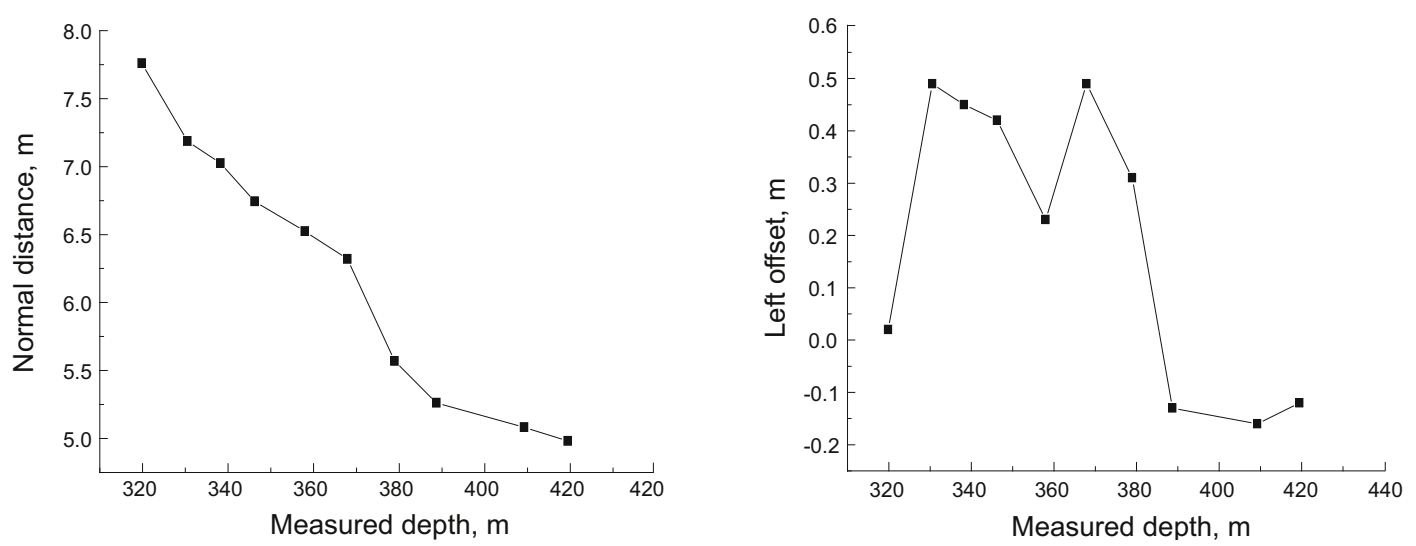

Fig. 13 The results of measurement and calculation in the build section of Well I

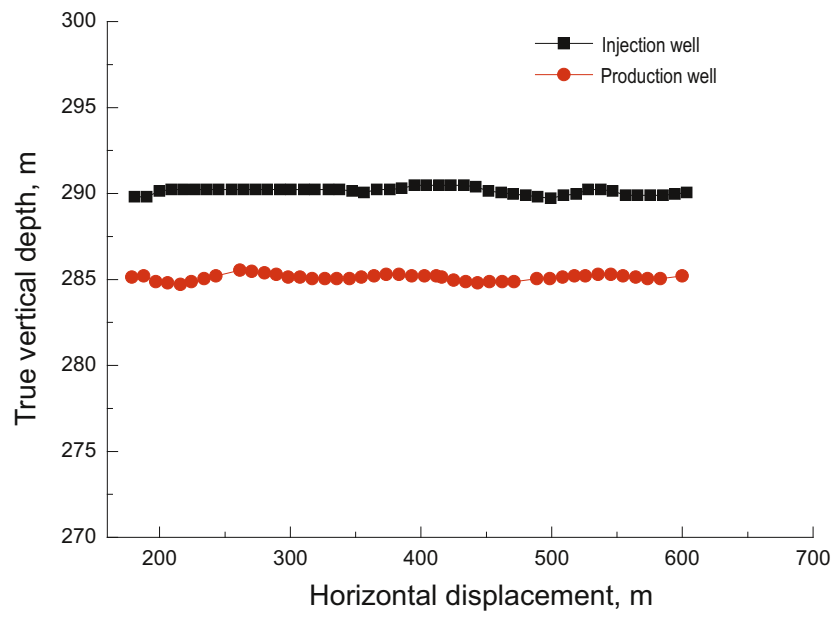

Fig. 14 Vertical projections of horizontal sections in the drilled Well I and Well P

\section{Conclusions}

1) Based on the ranging calculation method for the nonferromagnetic interference environment, the true directions and distances between two SAGD horizontal wells in a ferromagnetic interference environment can be effectively calculated by using the corrected data of the tri-axial alternating magnetic induction intensity detected by DDMGS. This meets the requirements of magnetic guidance while drilling SAGD horizontal wells.

2) Based on the detailed analysis of the detected signal characteristics, the high-precision narrow band Butterworth filter is designed to improve the signal quality. This filter can detect and collect the ultra-weak alternating magnetic signal in a noisy and ferromagnetic interference environment.

3) Because it can guide the directional drilling trajectory not only in the horizontal section but also in the build section, the DD-MGS can improve the quality and efficiency of directional drilling operations in SAGD horizontal wells.

\section{Acknowledgements}

The authors gratefully acknowledge the financial support from the Natural Science Foundation of China (NSFC, 51221003, U1262201). This research is also supported by other projects (Grant numbers: 2011ZX05009, 2013AA064803).

\section{References}

Al-Khodhori S, Al-Riyami H, Holweg P, et al. Connector conductor wells technology in Brunei Shell Petroleum-Achieving high profitability through multi-well bores and downhole connections. IADC/SPE Drilling Conference, 4-6 March 2008, Orlando, Florida, USA (paper SPE 111441-MS)

Bao Y, Wang J Y and Gates I D. History match of the Liaohe Oilfield SAGD operation-A vertical-horizontal well reservoir production machine. SPE Heavy Oil Conference Canada, 12-14 June 2012, Calgary, Alberta, Canada (paper SPE 157810-MS)

Corbett B. Steam assisted gravity drainage (SAGD) technologies and the oil sands. Alberta Chamber of Resources Directory. 1997: 30-36

Diao B B and Gao D L. A solenoid ranging while drilling guidance system. Acta Petrolei Sinica. 2011. 32(6): 1061-1066 (in Chinese)

Diao B B and Gao D L. Study of a ranging system based on dual solenoid assemblies, for determining the relative position of two adjacent wells. Computer Modeling in Engineering \& Sciences. 2013. 90(1): 77-90

Diao B B, Gao D L and Wu Z Y. Measurement of magnetic sub equivalent moment. Oil Drilling \& Production Technology. 2011a. 33(5): $42-45$ (in Chinese)

Diao B B, Gao D L and Wu Z Y. Magnet ranging calculation method of twin parallel horizontal wells steerable drilling. Journal of China University of Petroleum (Edition of Natural Science). 2011b. 35(6): 71-75 (in Chinese)

Dong J H, Wang X G, Qiao L, et al. Application of CBM multi-branch horizontal well for drilling technology in Fanzhuang Block. Coal Geology \& Exploration. 2008. 36(4): 21-24 (in Chinese)

Grace R D, Kuckes A F and Branton J. Operations at a deep relief well: the TXO Marshall. SPE Annual Technical Conference and Exhibition, 2-5 October 1988, Houston, Texas, USA (paper SPE 18059-MS)

Grills T L. Magnetic ranging technologies for drilling steam assisted gravity drainage well pairs and unique well geometries-A comparison of technologies. SPE International Thermal Operations and Heavy Oil Symposium and International Horizontal Well Technology Conference, 4-7 November 2002, Calgary, Alberta, Canada (paper SPE 79005-MS)

Gong Z M and Duan N Z. Technology of drilling mixed with gas for Lan M1-1 coalbed gas multi-branch horizontal well. Oil Drilling \& Production Technology. 2006. 28(1): 15-18 (in Chinese)

Hu H Y and Chen Q S. RMRS application on target-hitting of horizontal drilling. Geology and Prospecting. 2008. 44(6): 89-92 (in Chinese) 
Jones D L, Hoehn G L and Kuckes A F. Improved magnetic model for determination of range and direction to a blowout well. SPE Drilling Engineering. 1987. 2(4): 316-322 (paper SPE 14388-PA)

Kuckes A F, Hay R T, McMahon J, et al. New electromagnetic surveying/ranging method for drilling parallel horizontal twin wells SPE Drilling and Completion. 11(2): 85-90 (paper SPE 27466-MS)

Li C, Gao D L, Wu Z Y et al. A method for the detection of the distance $\&$ orientation of the relief well to a blowout well in offshore drilling. Computer Modeling in Engineering \& Sciences. 2012. 89(1): 39-56

Lin J, Song Z H, Luo Y H, et al. SAGD horizontal drilling technology. Xinjiang Oil \& Gas. 2009. 5(3): 56-60 (in Chinese)

Loparev D. Construction method of horizontal steam-injection and producing oil wells on the test sections OPU-4 and OPU-5 Yarega heavy oil deposit. SPE Russian Oil and Gas Technical Conference and Exhibition, 28-30 October 2008, Moscow, Russia (paper SPE 117378-MS)

Malik S, Zhang Y M, Asimi M A, et al. Steamflood with vertical injectors and horizontal producers in multiple zones. SPE Reservoir Evaluation \& Engineering. 2011. 14(2): 161-170 (paper SPE 129248-PA)

Mallary C R, Williamson H S, Pitzer R, et al. Collision avoidance using a single wire magnetic ranging technique at Milne point, Alaska. IADC/SPE Drilling Conference, 3-6 March 1998, Dallas, Texas, USA (paper SPE 39389-MS)

Mendoza H A, Finol J J and Butler R M. SAGD, pilot test in Venezuela. Latin American and Caribbean Petroleum Engineering Conference, 21-23 April 1999, Caracas, Venezuela (paper SPE 53687-MS)

Olberg T, Gilhuus T, Leraand F, et al. Re-entry and relief well drilling to kill an underground blowout in a subsea well: a case history of well 2/4-14. SPE/IADC Drilling Conference, 11-14 March 1991, Amsterdam, Netherlands (paper SPE 21991-MS)
Oskarsen R T, Wright J W, Fitterer D, et al. Rotating magnetic ranging service and single wire guidance tool facilitates in efficient downhole well connections. SPE/IADC Drilling Conference and Exhibition, 17-19 March 2009, Amsterdam, The Netherlands (paper SPE 119420-MS)

Qiao L, Shen R C, Huang H C, et al. Drilling technology of multibranch horizontal well. Acta Petrolei Sinica. 2007. 28(3): 112-115 (in Chinese)

Rach N M. New rotating magnet ranging systems useful in oil sands, CBM developments. Oil \& Gas Journal. 2004. 102(8): 47-49

Roes V C, Hartmann R A and Wright J W. Makarem-1 relief well planning and drilling. SPE Annual Technical Conference and Exhibition, 27-30 September 1998, New Orleans, Louisiana, USA (paper SPE 49059-MS)

Sun D K, Gao D L and Diao B B. The application of RMRS in the heavy oil \& ultra-heavy oil production. China Petroleum Machinery. 2011. 39(7): 73-76 (in Chinese)

Tarr B A, Kuckes A F and Ac M V. Use of a new ranging tool to position a vertical well adjacent to a horizontal well. SPE Drilling Engineering. 1992. 8(2): 128-134 (paper SPE 20446-PA)

Vandal B, Grills T and Wilson G. Rotating magnet ranging-A new drilling guidance technology. 8th One Day Conference on Horizontal Well Technology. Canadian Sections SPE/Petroleum Society, 2001

Vandal B, Grills T and Wilson G. A comprehensive comparison between the magnetic guidance tool and the rotating magnet ranging service. Canadian International Petroleum Conference, 8-1 Jun 2004, Calgary, Alberta, Canada

Yang M H, Xia H N, Qu S Y, et al. An MGT system applied to controlling accurate well tracks in SAGD twin horizontal wells. Drilling \& Production Technology. 2010. 33(3): 12-14 (in Chinese)

(Edited by Sun Yanhua) 